\title{
Benefits of Delay Tolerant Networking for Earth Science Missions
}

\author{
Faith A. Davis \\ Microwave \& Communication \\ Systems Branch \\ NASA/GSFC Code 567.3 \\ Greenbelt, MD 20771 \\ 301-286-5578 \\ faith.a.davis@nasa.gov
}

\author{
Jane K. Marquart \\ Flight Software Branch \\ NASA/GSFC Code 582 \\ Greenbelt, MD 20771 \\ 301-286-7851 \\ jane.k.marquart@nasa.gov
}

\author{
Greg Menke \\ Columbus Technologies \\ Greenbelt, MD 20771 \\ 301-286-4127 \\ Gregory.d.menke@nasa.gov
}

\begin{abstract}
To date there has been much discussion about the value of Delay Tolerant Networking (DTN) for space missions. Claims of various benefits, based on paper analysis, are good; however a benefits statement with empirical evidence to support is even better. This paper presents potential and actual advantages of using DTN for Earth science missions based on results from multiple demonstrations, conducted by the Communications, Standards, and Technology Laboratory (CSTL) at NASA Goddard Space Flight Center (GSFC). Demonstrations included two flight demonstrations using the Earth Observing Mission 1 (EO-1) and the Near Earth Network (NEN), a ground based demonstration over satellite links to the Internet Router in Space (IRIS) payload on Intelsat-14, and others using the NASA Tracking Data Relay Satellite System (TDRSS). Real and potential findings include increased flexibility and efficiency in science campaigns, reduced latency in a collaborative science scenario, and improved scientist-instrument communication and control.
\end{abstract}

\section{TABLE OF CONTENTS}

1. INTRODUCTION ....................................................... 1

2. EO-1 EXPERIMENT .......................................... 2

3. IRIS EXPERIMENT ............................................... 4

4. TDRSS EXPERIMENT ...................................... 7

5. SUMMARY.......................................................... 10

6. FUTURE WORK ................................................. 10

REFERENCES.......................................................... 10

BIOGRAPHY.......................................................... 11

\section{INTRODUCTION}

The Delay Tolerant Network (DTN) protocol is typically associated with Deep Space missions where communication links are very slow, have large delays, and can often be intermittent. Being a store and forward protocol, it is also assumed to only be applicable in a multi-hop environment. However, it is the premise of this paper to show, through demonstration, the applicability of DTN to other types of environments, particularly Low Earth Orbit, Earth Science, and single spacecraft missions. Potential benefits were explored using flight, ground based over satellite links, and high-fidelity lab demonstrations. Sensor webs, operations, and asymmetric links are areas which displayed potential benefits to missions with DTN usage.

Sensor Webs: According to NASA's Direction 2005 \& Beyond, "NASA will develop new space-based technology to monitor the major interactions of the land, oceans, atmosphere, ice, and life that comprise the Earth system. From there, researchers envision an intelligent and integrated observation network comprised of sensors deployed to vantage points from the Earth's subsurface to deep space. This 'sensor web' will provide timely, ondemand data and analysis to users who can enable practical benefits for scientific research, national policymaking, economic growth, natural hazard mitigation, and the exploration of other planets in this solar system and beyond." [1] The communications infrastructure must evolve in order to support NASA's vision of an integrated observation network. Missions typically plan their communications system with a single mission in mind, their own. Each mission should be viewed as a node in the integrated observation network to be able to enjoy the practical benefits that sensor web scenarios would afford.

The IRIS collaborative sensor web demonstration focused on the benefits of networking as it relates to areas such as autonomous alert mechanisms between nodes, improved data collection and measurement precision, event synchronization, and onboard and ground agent synchronization. Rapid response and distribution of information is critical. Autonomous planning and data transmission via use of multiple downlink access points is required. The manual configuration of sensors as they continue to expand will become unmanageable and operations costs will surely grow. During this demonstration, the store and forward network approach of DTN and the routing capability minimized the latency between nodes by automatically routing data packets to their destination despite intermittent links.

Improved Operations: The EO- 1 spacecraft is an Earth imaging observatory with a multispectral land imaging instrument onboard that is a significant improvement over the Landsat 7 ETM+ instrument. At the end of its science mission, NASA Headquarters approved a plan for an Extended Mission operations phase, where new technologies could be validated. Current EO-1 operations require coordination of data transfers across the space link with the scheduled ground contacts. This is done manually. DTN enables the decoupling of these two activities by allowing onboard data to be held in persistent storage until a contact is available, at which time it is automatically sent to the Command and Data Handling (C\&DH) for downlink.

Because of the large volume of hyperspectral data, it is typically down linked at very high rates. The data are stored 
at the ground station for future transmission to the user via terrestrial links, or in some cases, physical media. Using DTN at the ground station, data can autonomously flow to the user as the link permits, removing the need for manual intervention. On the forward link, data to be uplinked can be sent to the ground station at any time and remain in the ground network until the forward link becomes available.

Asymmetric Links: As Earth Science instruments become more advanced, the volume of data generated onboard significantly increases. This in turn requires high rate downlinks to ensure that all data is returned to the user on Earth. Spacecraft avionics currently support hundreds of megabytes of downlink capacity between the spacecraft and the ground stations. The forward link between the ground station and the flight systems however, do not support the same level of throughput.

In the CSTL a test was set up to emulate the conditions of a multiple access (MA) user and a S-band single access (SSA) user of Tracking Data Relay Satellite (TDRS) with asymmetric and simplex links. This was done with actual TDRS time. The store and forward capability of DTN as well as the networked architecture that supports endpoint addressing, enables autonomous routing of data when links become available, to any user. The rate buffering of DTN allows data to be sent at rates conducive to the network.

The rest of the paper is organized into individual experiments and the benefits observed. Section 2 describes the EO-1 demonstration. Section 3 describes the IRIS collaborative science demonstration and Section 4 describes the TDRS. Section 5 summarizes the findings and discusses benefits for earth scientists. Finally, future work is described in Section 6.

\section{EO-1 EXPERIMENT}

Goddard Space Flight Center successfully demonstrated an on-obit DTN node using the EO-1 satellite on December 8102010 and February 8-10, 2011 as part of a multi-phase demonstration activity sponsored in part by the Delay Tolerant Readiness Project, NASA HQ. The general goal of the EO-1 demonstration activity was to validate DTN technology for Earth Observing and other Low Earth Orbit (LEO) missions in order to:

1. Understand the implications of implementation for flight and ground systems.

2. Demonstrate benefits of the technology.

3. Discover new approaches and uses for the DTN technology.

In addition, and specific to EO-1, this demo was expected to recover, via DTN, real-time housekeeping telemetry that has been lost due to a hardware failure in 2006 .

Among the potential DTN benefits identified for EO-1 were:

- Decoupling the communication scheduling from command \& telemetry generation. Current EO-1 operations require manual control and coordination of uplink and downlink data transfers to coordinate data transmission with scheduled uplink and downlink ground station contacts. The DTN bundle protocol provides "persistent storage" allowing data to be held within the network pending future data link contacts. This enables the decoupling of data generation from contact schedules. To demonstrate this capability, the test plan included the transfer of contact schedules, during non-contact periods, from the Science Operations Center (SOC) to EO-1.

- Recovery of C\&DH housekeeping data. Since 2006, the EO-1 Housekeeping data stored between ground contacts has been lost due to a failure in the (C\&DH) Solid State Recorder (SSR). Using DTN "persistent storage" and the priority function, the plan included recovery of some housekeeping packets.

- $\quad$ Provide automated store and forward of satellite data. As mentioned earlier, hyperspectral and other imaging satellites produce large volumes of data. Typically, the data is transmitted to ground stations at very high data rates (i.e. $150 \mathrm{Mbps}$ ). The data is usually captured at the ground station and forwarded later at a lower rate via terrestrial data links or physical media such as data tapes. This activity demonstrated the DTN capability to automatically and reliably store and forward satellite data via custody transfer between the spacecraft and the Mission Operations Center (MOC).

The demonstration included three phases executed over a total of $6 \frac{1}{2}$ days, of which $3 \frac{1}{2}$ were in December, and 3 days were in February. Altogether there were 14 contacts available with approximately six to seven minutes of each pass available for testing. Only one contact was via the Wallops Ground Station (WGS). Phase 1 was the initial integration of the DTN code into the EO-1 onboard software and the EO-1 MOC. The goal was to verify the basic communications function of receiving/sending DTN bundles, and contact schedules, between the flight and ground MOC DTN nodes. Data exchange included both ASCII text and files. This also provided insight into infusion of DTN into existing flight and ground systems. Phase 2 tests were designed to demonstrate the automated store and forward benefit using the custody transfer and priority functions of DTN within a three node network. As a side benefit particular to EO-1, recovery of EO-1 housekeeping data were also included. This phase provided insight into potential benefits of the DTN technology. Phase 3 tests were a repeat of Phase 2 but with the addition of a DTN node WGS.

This created a four node network and gave insight into infusion of DTN into ground station operations. The full network configuration is illustrated in Figure 1. 


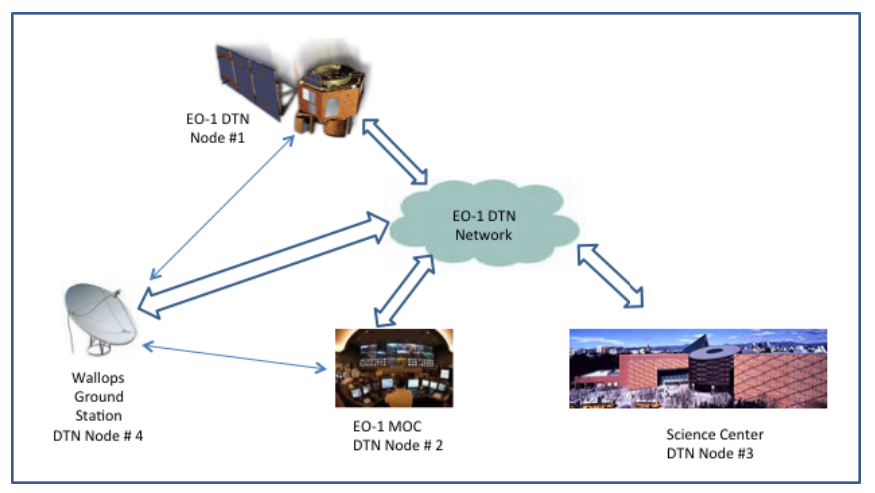

Figure 1: EO-1 Demonstration Configuration

Data Flow and Protocols

All EO-1 DTN network nodes use the Bundle Protocol (BP) at the network layer. However, different transport layers were used. For the space to ground link, EO-1 and the MOC implemented Licklider Transport Protocol (LTP). LTP provides reliable transfers point to point from the EO-1 Flight DTN node to the MOC DTN node. LTP segments were sized to fit within the data field of the existing EO-1 data link layer units, which are Consultative Committee for Space Data Systems (CCSDS) Advanced Orbiting Systems (AOS) frames on the downlink. For the uplink, the LTP segments were inserted in the CCSDS command packet data field. In Phases 2 and 3, the Transmission Control Protocol (TCP) was the transport protocol of choice for all ground links. TCP provides a reliable transport between the EO-1 $\mathrm{MOC}$ and the Science Operations Center (SOC), as well as the WGS MOC and EO-1 MOC. The protocol stacks are illustrated in Figure 2.

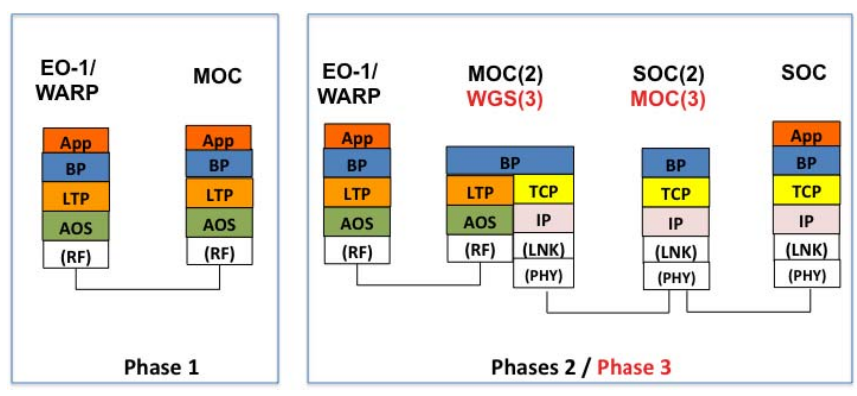

Figure 2: EO-1 Protocol View

\section{Test Results}

Phase 1 verified the communication links as well as some functionality of DTN. Impact to operations was also observed. In particular, ground commands, text strings, and files were transferred between the flight node and the mission operations center. During the file transfer test (from flight node to ground node), the file was received by the MOC DTN node despite the fact that only $16-20 \%$ of the LTP segments were forwarded from the ground front-end equipment to the MOC DTN node. This was enough to complete the file transfer and illustrated the benefit of DTN as compared to file transfer without DTN, where the file transfer would have been incomplete. It also gave insight into the consequential LTP acknowledgement (ACK) traffic on the forward link.

Phase 2 demonstrated the ability of DTN to store and forward housekeeping data. This capability 'conceptually' eliminates the need for an onboard housekeeping solid state recorder, since data is persistently stored until custody of data is accepted by another DTN node. It also incorporated the third DTN node at the science operations data center. Contacts were either manually started or uploaded as file transfers at certain points throughout the test. Phase 2 targeted the benefits defined earlier including:

Decoupling of schedule and telemetry/command: To demonstrate this capability, the test plan included the transfer of contact schedules, during non-contact periods, from the EO-1 SOC to the EO-1 spacecraft. The schedule was transferred autonomously without operator intervention and was executed on-board and on the ground DTN nodes to control link availability. On the downlink, EO-1 housekeeping data was transferred to the MOC. Housekeeping data were collected during the non-contact period and prioritized on the downlink once a contact began. No support from operations personnel was required.

Recovery of Housekeeping data: During ground contact, the data generated is called "real-time". This real-time housekeeping data are sent by the formatting application as higher priority, while the housekeeping data collected during the non-contact time are sent with a lower priority. This demonstrated the DTN data prioritization capability and the recovery of 'out of contact' stored housekeeping data.

Automated Store and Forward: Specifically, the contact schedule file was transferred from the SOC DTN node through the MOC node to the Spacecraft node using custody transfer. Custody transfer enabled the SOC to send data to the spacecraft (nominally this would be the science applications) outside the contact period. Quality of Service (QOS) specified both forwarding and delivery confirmation of the data.

Phase 3 incorporated a fourth DTN node at the Wallops Ground Station. This node simulated a Ground Station DTN node that facilitates data transfer with the flight DTN node to demonstrate custody transfer capability. The concept for the Wallops ground station is to "tee" off the Sband telemetry stream and send to a co-located DTN Workstation. This DTN node parses the incoming telemetry stream and extracts and processes LTP data segments. It is also connected to the MOC ground system machine to facilitate uplink messaging with the flight DTN node. Phase 3 was mostly successful. The ability to initiate a contact via DTN command from the Wallops DTN node verified the forward link communications. The Wallops DTN node successfully received telemetry (in the form of CCSDS Virtual Channel Data Units or VCDUs) from Wallops Packet Telemetry Processor (PTP) and successfully extracted the packets. However, it did not recognize them as LTP packets so did not pass these along to the DTN software. Therefore, the Wallops DTN node did not process any incoming LTP data from the spacecraft, so no 
Housekeeping packets or ACKs or CUSTODY TRANSFER messages were processed by the Wallops DTN node. Since there was only one contact within the test period that included Wallops, there were no more opportunities to fix and retry this test. Post-demo analysis, however, revealed the problem to be a software bug in the support software. Once corrected, the flight data were replayed through the ground system elements and the data were successfully received by the EO-1 MOC and SOC.

\section{Summary}

The principal DTN test objectives were demonstrated and provided the beginnings of answers to the benefits of the protocol to Earth Science missions. BP/LTP worked not only in the nominal or expected conditions, but also enabled the receipt of a file, which would otherwise have been lost, during unexpected anomalous conditions. Store and forward, as well as custody transfer were clearly beneficial for autonomous operations. Integration of DTN into the Wallops Flight Facility Ground Station was extremely simple and straight-forward due to the well-defined interfaces of the ground station equipment and the front end processor. Using standard protocols for these interfaces was key. When bundles were expected but not received, the operations and test team felt a lack of insight into the flight DTN software. Given the lack of DTN status telemetry, it was difficult to know what was going on or where the problem lay. Improvement in this area would be beneficial to mission operations acceptance, but this is easily corrected with enhancement of the protocol code. The use of DTN in the recovery of EO-1 telemetry clearly shows beneficial side-effects and an unexpected use of the technology.

Security and firewall problems occur if there is a need to go outside the mission network. Waivers were needed to send data from the EO-1 MOC to the SOC due to the fact that they were on different networks and thus had different policies. Depending on the configuration of the network(s) involved, this may be a "long-lead" item.

As mentioned earlier, this demo was subject to the constraints of all flight software and thus had to adhere to policy and system engineering requirements. Policy requirements may include things such as: specifying one telemetry packet which contains the spacecraft-to-MOC DTN data unit; specifying one command packet which contains the MOC-to-spacecraft DTN data unit; specifying one command packet which contains administrative commands sent to the spacecraft DTN by the MOC; or limiting transmission of DTN data unit packets to an interval of given length and which begins when the Flight Operations Team authorizes the start of transmission. System engineering requirements were met by using DTN's LTP protocol to set a maximum return and forward link utilization. LTP provides several useful side effects, including reliable bundle transfer and segmentation/packing of large or small bundles into packets sized for convenient transfer through the normal command/telemetry channels.

Overall, insight was gained given the limited amount of actual test time on the EO-1 spacecraft, and because the planned tests encountered some obstacles. Information regarding anomalous situations was gathered as well. A window into future benefits was opened. These may include increased flexibility for onboard product generation. The current interface mechanism between the ground agent and the onboard science software requires significant overhead in terms of onboard processing, ground processing, and overall systems engineering. Due to the constraints of the Sband link/bandwidth, considerable effort is spent performing systems engineering of onboard analysis and onboard generated products. Operational use of DTN would free the software from micro-managing this data stream by automatically managing data custody and prioritization. It should be noted that for this demo, the DTN code was not fully integrated with the existing science application code; rather it took the place of the science application software. This was due in part to resources available for the demo, as well as a risk-reduction to prove the technology without impact to the spacecraft.

One final observation that aligns with the IRIS demo described later in the following section is the potential for increased flexibility for sensor web applications development. All of the, ten or more, sensor web campaigns demonstrated by EO-1 rely on static, pre-developed campaigns requiring ground personnel to identify the source/trigger and response assets in the sensor web. An onboard DTN node could enable EO-1 to participate in a publish and subscribe fashion with nodes dynamically added to the DTN. This opens up a whole range of possible triggers and response assets that only need publish alerts to the sensor web DTN network. Any node subscribing to the relevant class of messages can then respond, enabling a true sensor web paradigm of information sharing and enhanced earth observation.

\section{IRIS DEMONSTRATION}

Goddard Space Flight Center demonstrated a collaborative earth science sensor web scenario in the Communications, Standards, and Technology Lab (CSTL), utilizing Cisco's IRIS payload on Intelsat-14. IRIS is the first router in geosynchronous orbit and served as an IP relay in this sequence of events. The primary objective of this demonstration was to examine advantages of Bundle Protocol (BP) and Internet Protocol (IP) relay use in collaborative science missions. The thought being that automated storing, forwarding, and routing functions within a network would reduce the latencies between collaborative science assets. 


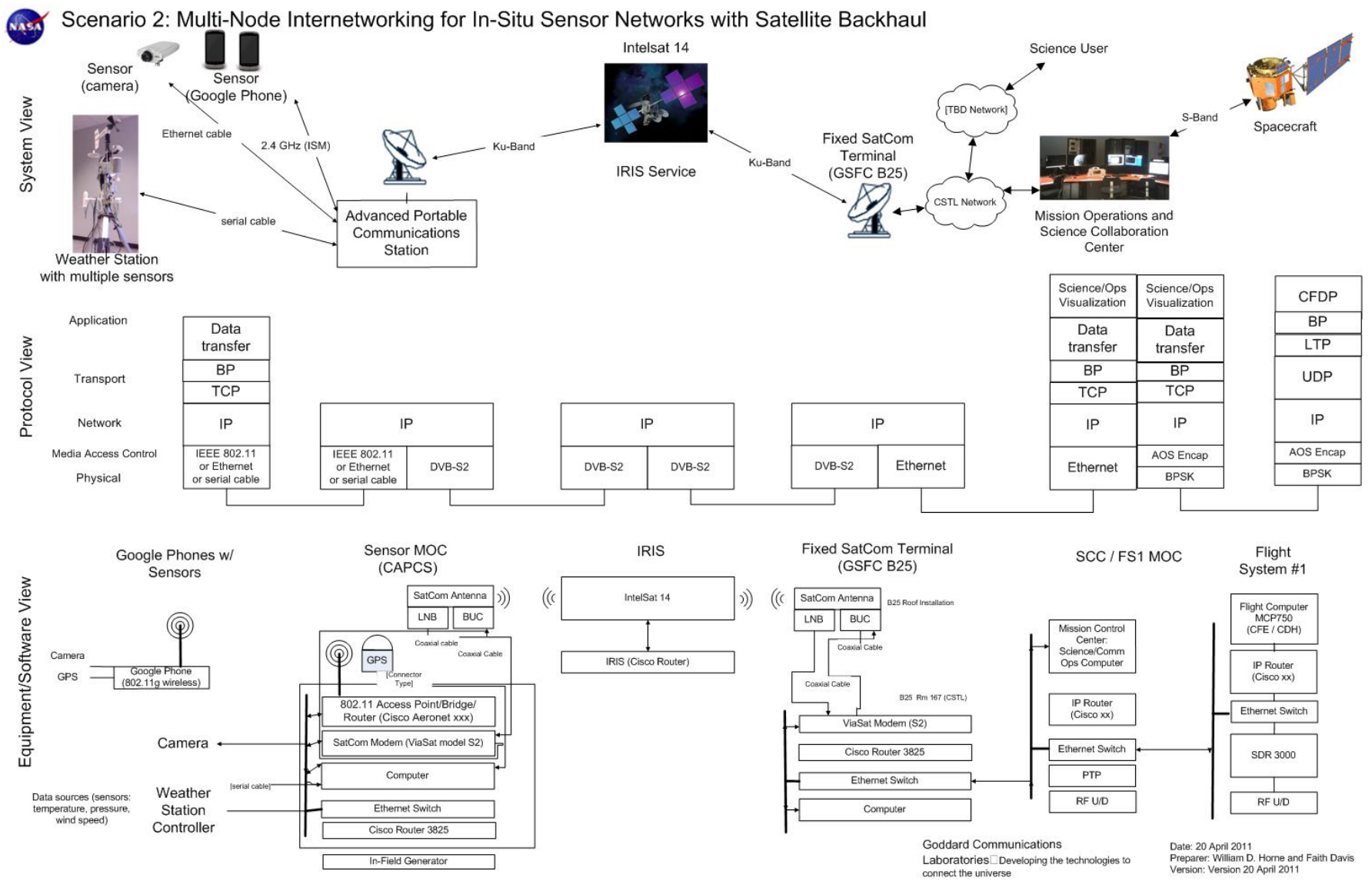

\section{Figure 3: IRIS Collaborative Science Demonstration}

The details of the test scenario are depicted in Figure 3. The data flow is from right to left. It shows an earth science mission spacecraft, Flight System 1 (FS1), transferring bundles containing data, such as event alerts, to in-situ sensors on the ground. In this instance, a bundle directs the in-situ sensor to take a look at an interesting event observed from Flight System 1. The data are forwarded at each node, from Flight System 1 to the FS1 Mission Operations Center (MOC), then to the Science Collaboration Center (SCC), via IRIS to the Sensor MOC. The Sensor MOC forwards the data to the sensors, which is the desired action to perform. The Sensor MOC in Figure 3 is also known as CSTL Advanced Portable Communication System (CAPCS). If any link is disrupted, the data are stored at each node until the link becomes available. Bundles were transferred with a nominal data rate of approximately $170 \mathrm{Kbps}$. Higher peak rates were seen when the IRIS radio frequency link was reconnected and queued bundles flowed at the maximum achievable rate.

Data observed from the sensors are then sent back to the Science Collaboration Center (SCC). At the SCC several activities take place. The in-situ sensor data are written to SCC disk storage for processing by ILIADS. ILIADS is a visualization software package that is acting as a piece of ground support equipment and a facet of the overall collaboration model. Additionally, the sensor data may be combined with the primary observation data from Flight System 1 for collective science applications. Or perhaps
Flight System 1 could enhance its operation based on measurements received from the in-situ sensors.

In the CSTL the scenario was executed with science "events" being generated asynchronously by toggling a switch on the Flight System 1 (FS1) "flight computer", which in turn generated the event bundles. When a link between the Flight System 1 and FS1 MOC was unavailable, these event bundles are queued on Flight System 1. Over a period of forty-three minutes and one second, when a link was available, these event bundles were down linked from Flight System 1, forwarded via IRIS to CAPCS, and then on to the sensors. In this demonstration, the in-situ sensors are Android Google phones, a collection of weather sensors, and a camera.

In order to emulate a variety of links with different contact periods, the link between Flight System 1 and the MOC is varied on for five minutes, off for five minutes, then on for ten minutes. The contacts were driven by the flight software scheduler, which was programmed with the contact duration and interval between contacts. The flight software scheduler controlled both the BP/LTP contact graph routing parameters and the CCSDS File Delivery Protocol (CFDP) file transfer enable/disable. The IRIS link was disrupted twice during the test. This was done by turning off power to the modem in the portable terminal. When the IRIS to CAPCS link was unavailable, bundles were stored in the SCC until the next available contact. 
In response to the event bundles received from Flight System 1, bundles containing GPS coordinates were sent from the Google phones back to Flight System 1. Hence, the rate of bundles sent from the Android Google phones varies. The bundles originating at the Google phones also experienced disruptions of the IRIS link and FS1 MOC to Flight System 1 link. Bundles are queued in the Sensor MOC when the IRIS link is down. Bundles are queued in the SCC when the FS1 MOC to Flight System 1 link is unavailable. The bundles sent from the Google phones to CAPCS back to the science collaboration center were sent using Bytewalla, an implementation of DTN2 for Android.

Throughout the entire demonstration, weather station data and camera images are sent multicast back to SCC and to the node in Raleigh, North Carolina. The weather station is sending pressure, temperature, wind speed, measurements throughout the duration of the test. The weather station data are sent using user datagram protocol (UDP) Multicast. The weather station sends an approximately 630 Byte weather report bundle every 5 seconds. The camera sends an approximately 32 Kbyte JPEG bundle every 3 seconds. There was an additional data stream throughout the test between the Voice Over IP (VOIP) phones connected to CAPCS and the SCC. This data is sent using Real Time Protocol (RTP).

There is also a VOIP phone associated with CAPCS and the FS1 MOC. This was used randomly throughout the test to perturb the protocol mix and add complexity to the test.

It should be noted that BP/TCP was used for the space link between IRIS and the ground centers due to the fact that there was no expectation of maximum link utilization. For maximum link utilization on space links, LTP would be the logical choice.

\section{Results}

A collaborative science sensor web can be done without an IP relay, like IRIS; however, it would not be as efficient. Figures 4 and 5 compare data flows using IRIS, without routing capabilities, as the backhaul between the Sensor MOC and the SCC and using IRIS as an IP relay. Solid lines in the diagram refer to the data path with an IP relay and dashed lines refer to the data path without an IP relay. For clarity, Figure 4 illustrates the data path from the Flight System to the Sensor MOC while Figure 5 displays the data path of in-situ sensor data from the sensors to the SCC.

In Figure 4, data flows from Flight System 1 to FS1 MOC then to the SCC. These two steps, (1) and (2), are the same with or without an IP relay. The differences are seen from step (3) onward. If Flight System 1 wants to send its data to both the node at RTP and the Sensor MOC it can do that via multicast with an IP relay. From the SCC, the data would go to IRIS (step (3)) and then to both North Carolina Research Triangle Park (RC NTP) (4) and the Sensor MOC (4) simultaneously. So, data is sent with an IP relay from Flight System 1 to RC NTP and the sensor MOC in four steps. Without an IP relay, multicast capability is not available so the path from SCC to both NC RTP and the sensor MOC changes. From SCC the data must go to IRIS via KU1 band (3) to Sensor MOC (4). The same data must also go from SCC to NC RTP node via KU2 band $(5,6)$. Hence, the data path without an IP relay requires two additional hops.

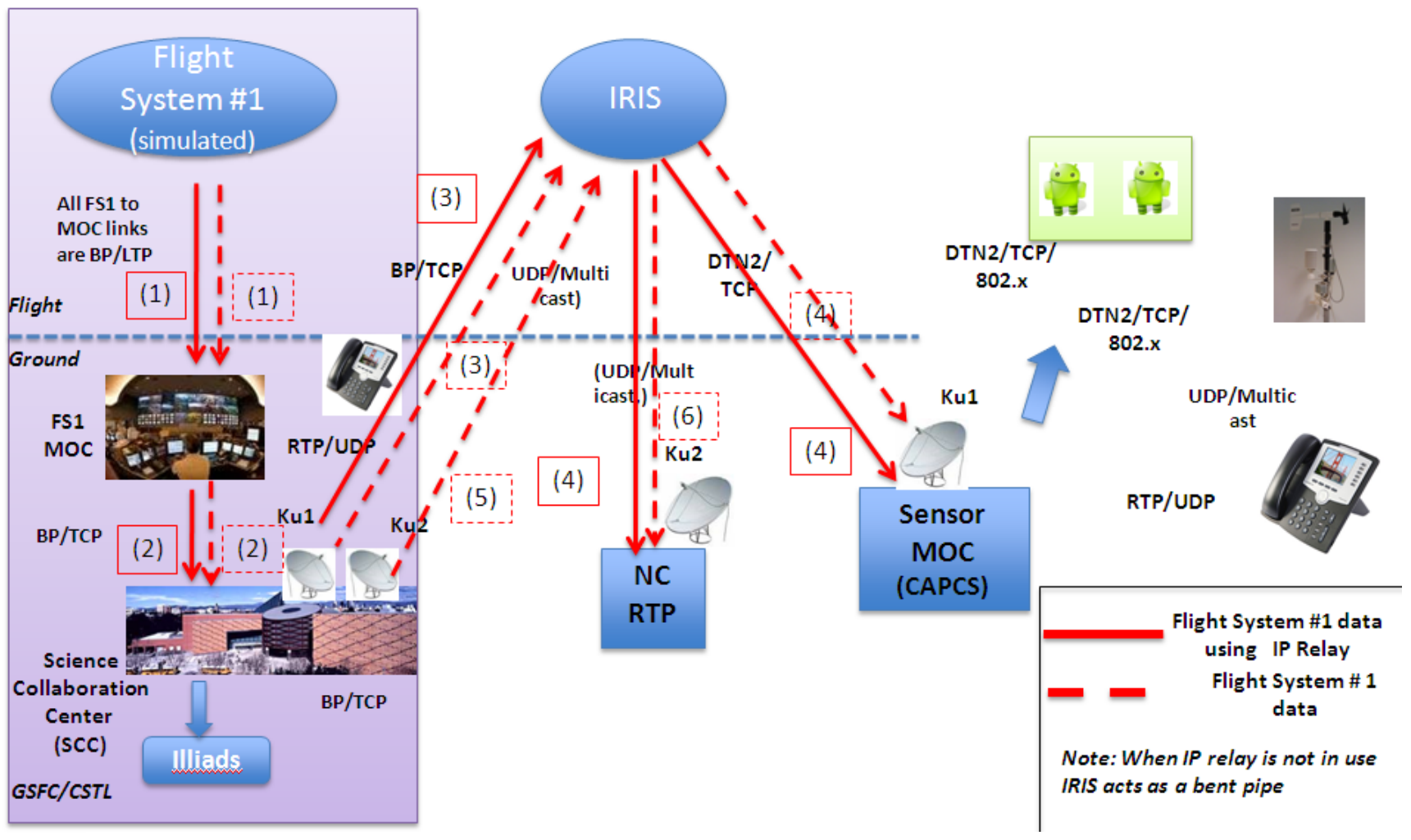

Figure 4: Path of Flight System \# 1 data for IRIS Scenario with and without IP relay 


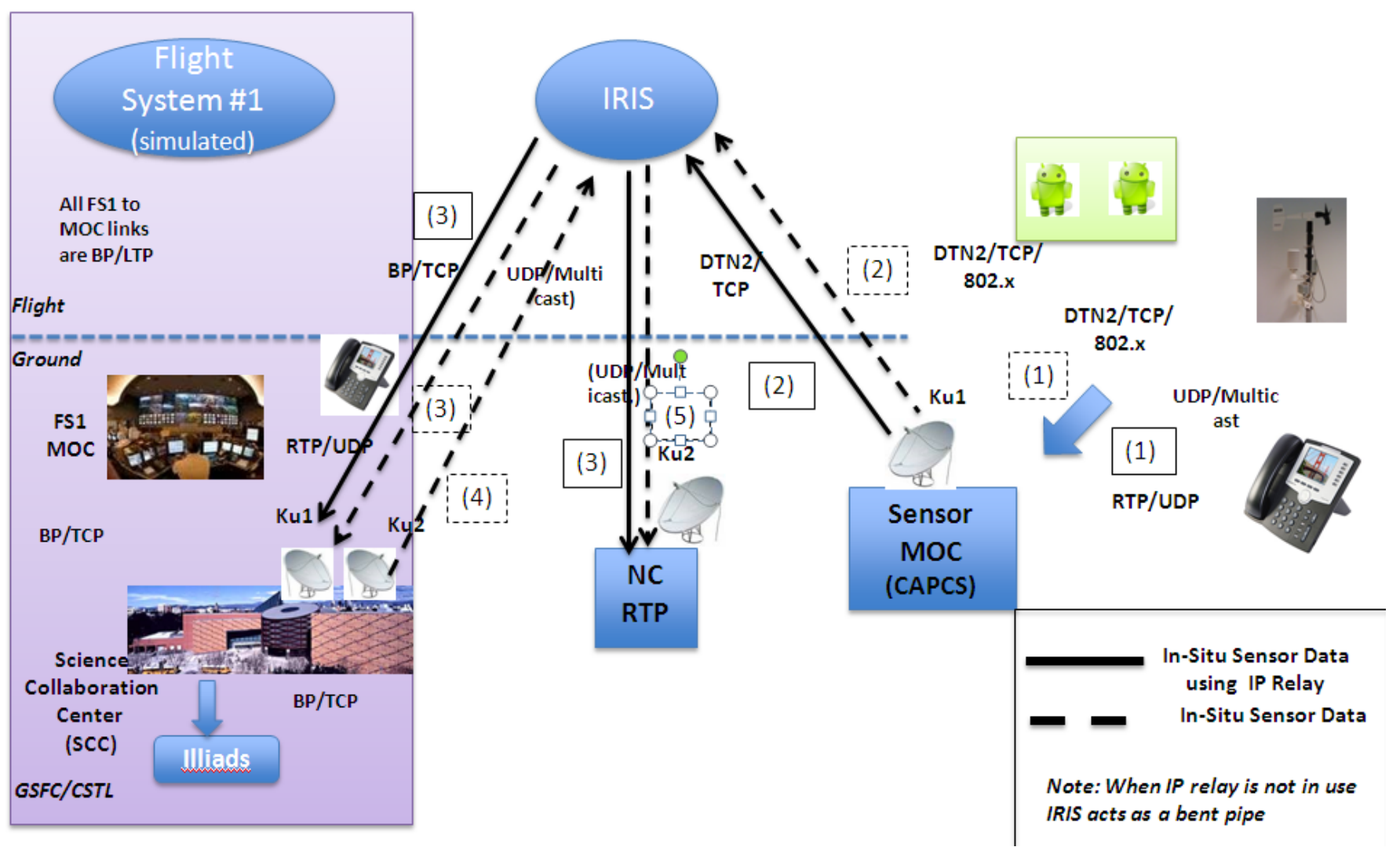

\section{Figure 5: Path of In-Situ sensor data for IRIS scenario with and without IP relay using TDRS satellite}

In figure 5, the node at RTP and the sensor MOC are also on two different frequency bands, $\mathrm{Ku} 1$ and $\mathrm{Ku} 2$. A non relay satellite transponder does not support cross beam communication. However, this functionality is available with a router in space. Using the IRIS, the weather station and Google phone data would go to the Sensor MOC (1) and then directly to both RTP and SCC using multicast $(2,3)$, taking a total of three hops. This is shown with solid black lines in the Figure 5. In order to get weather station data to both RTP and the SCC node without cross beam communication, the weather station data would have to travel the same route from Sensor MOC (1), then to SCC via IRIS Ku1 $(2,3)$ to get to the SCC Node, but would then have to be sent back up on Ku2 beam via IRIS (4) to get to the RTP node (5), resulting in an additional two hops and a delay of two round trip times. This is illustrated in Figure 5 with the dashed black lines.

\section{Summary}

Results indicated potential advantages of using Bundle Protocol for a collaborative earth science mission. This scenario allowed the latency associated with sensor web alerts and collaborative science to be assessed. Nodes were able to store and forward event data in the presence of disrupted links without data loss and without operational intervention. The latency between the event detection at Flight System 1 and notification at the sensors was small. The IP relay reduced the number of hops necessary to communicate between the nodes collaboratively, which in turn helped to improve the overall latency in the system. Multicast data transfer and cross-beam communication were also demonstrated via the IP relay. While real-life mission results will vary depending on the number of nodes and contact periods, this demonstration clearly showed the potential for DTN to enable an environment where alerts are autonomously "published" throughout a web system and users "subscribe" to the alerts/events and take action accordingly. The autonomous store and forward capability of DTN along with a networked architecture enables the science community to receive alerts quickly and respond accordingly.

\section{TDRSS DEMONSTRATION}

As part of the DTN Readiness Program, GSFC performed several DTN/IP protocol communication tests with the TDRSS spacecraft. The goal of these tests was to observe how the internetworking protocols, namely the Bundle Protocol (BP) and the Internet Protocol (IP) perform when communicating via asymmetric TDRSS links with unidirectional periods. This is a typical Earth Science/Low Earth Orbit (LEO) mission configuration. The CSTL was set up to emulate the conditions of a multiple access user and a S-band single access user of TDRS. Both asymmetric and intermittent simplex links were exercised. 

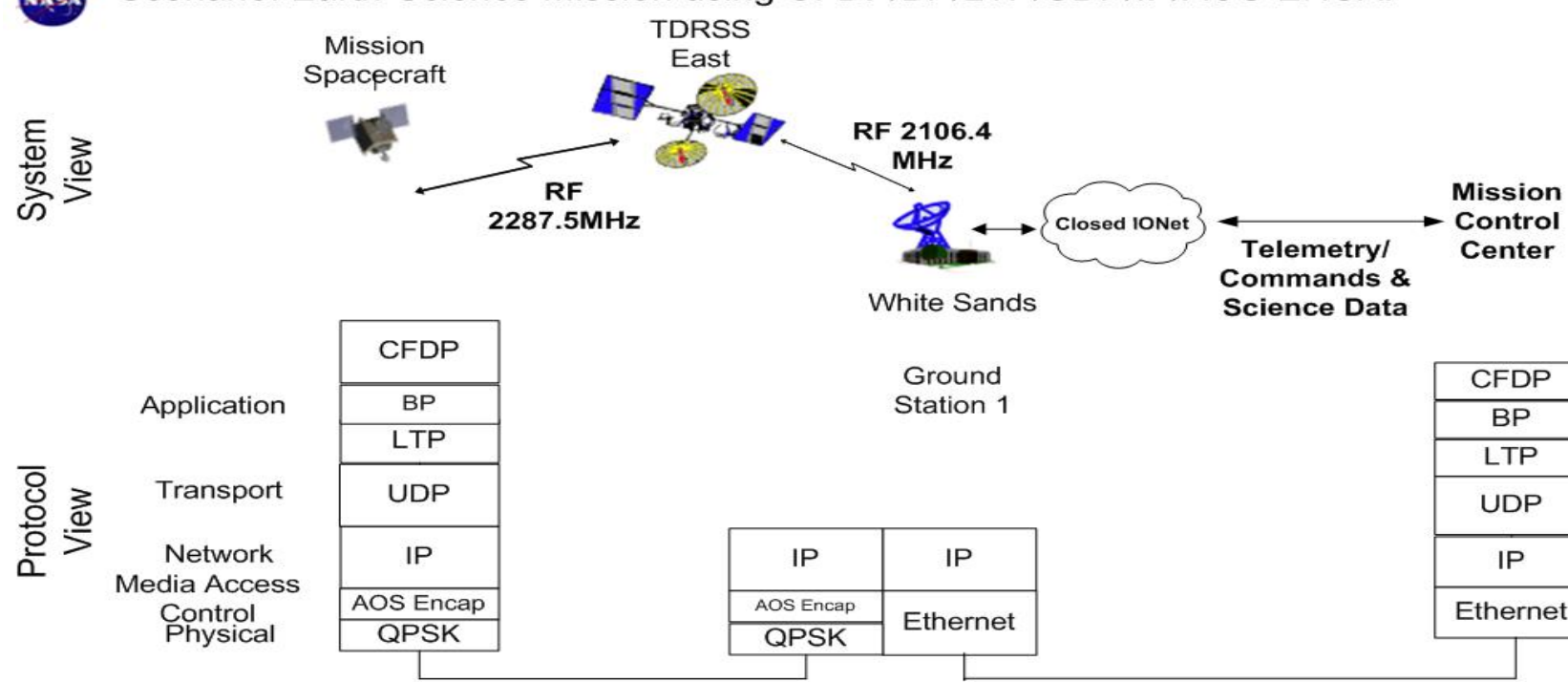

Ground Station 1 Science Data

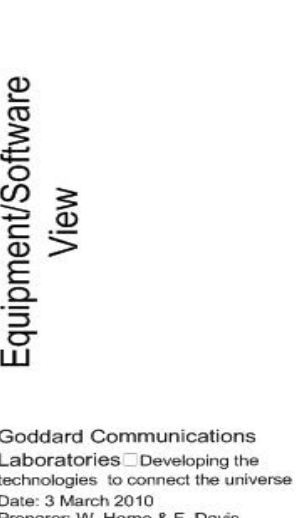

FS1

\begin{tabular}{|c|c|}
\hline IP & IP \\
\cline { 1 - 1 } AOS Encap & \multirow{2}{*}{ Ethernet } \\
\hline QPSK & \\
\hline
\end{tabular}

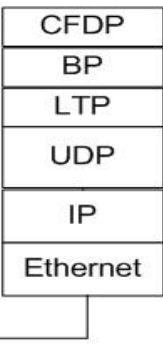

GS1

MCC

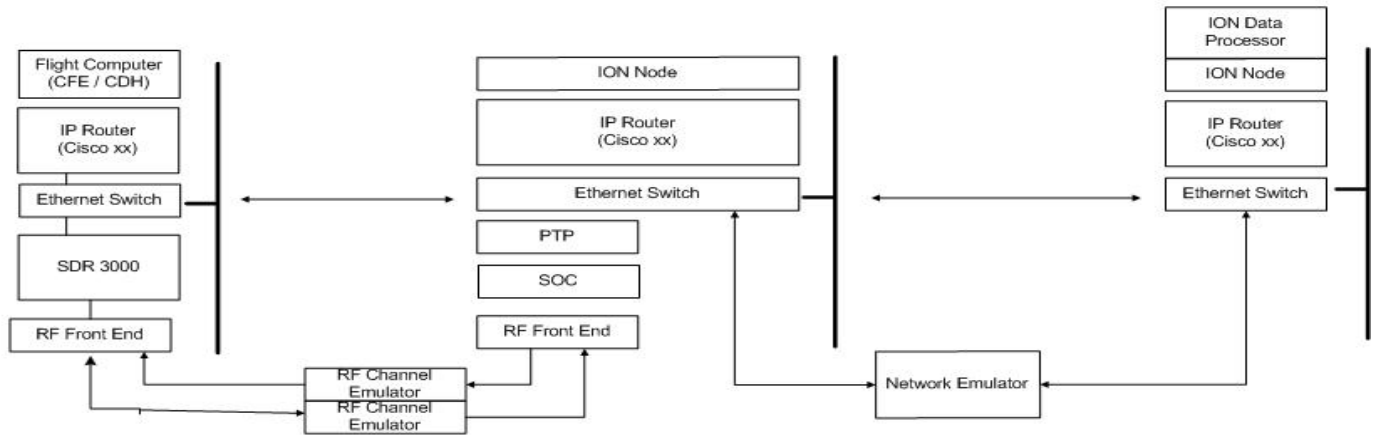

Figure 6: TDRS Demonstration Scenario

There were three network nodes: the spacecraft, the ground station, and the mission control center. Files were transferred, via CFDP, between the spacecraft and the mission operations center using the TDRS link. This test scenario is executed with the protocol stack, CCSDS File Delivery Protocol (CFDP) Class 1/BP/LTP/IP/AOS Encap, which is depicted in Figure 6. CFDP Class 1 provides unreliable file transfer. BP optionally uses "custody transfer" for its data units to supply reliable transfer. CFDP class 1 was selected for the BP/LTP stack since LTP already ensures the reliable transfer of files. Class 1 CFDP and LTP would be redundant. CFDP was configured such that the retry count was never exhausted. The details on the test scenario are depicted in Figure 6. This is an earth science mission spacecraft that is transferring a 1.6 Megabyte file to the ground via TDRS East. A 1.6 Megabyte file was arbitrarily selected so that on the $128 \mathrm{kbps}$ link a reasonable amount of data points could be captured for each file transferred. The file is then forwarded on to the Mission Control Center (MCC). The file is transferred via asymmetric TDRSS links with uni-directional periods.
Results

The graph in Figure 7 is a sample of what was observed during the TDRS testing. The graph is depicting a MA TDRSS user with asymmetric data rates and simplex links. The return link is $64 \mathrm{Kbps}$ and the forward link is $18 \mathrm{Kbps}$. The period begins with return link only followed by an intermittent forward link for the remainder of the test. From $t$ $=0$ through $\mathrm{t}=825$, the forward link is down. File segments are delivered until the maximum outstanding queue depth is reached. At 825 seconds, forward link is started, the protocol handshakes complete, and transfers resume. The erratic rx-data rate seen after 850 seconds is unexplained and so far unduplicated.

There was an anomaly observed with LTP that could not be duplicated in follow-up testing. The LTP state machine did not resume nominal operation when the radio link transitioned from return link only to full duplex. The LTP handshake for the outstanding transfers did not occur and the ground-side LTP engine was "stuck" not acknowledging the outstanding transfers, which held up the "spacecraft" sender from starting new ones. However, this stack also successfully transferred files when the TDRSS link was replaced with a land-line link. So the anomaly may well have been a configuration issue and not a protocol issue. 
(tdrss2) T, Ray CFDP Class1 over ION/LTP/UDP/IP, 64kbps, fdx

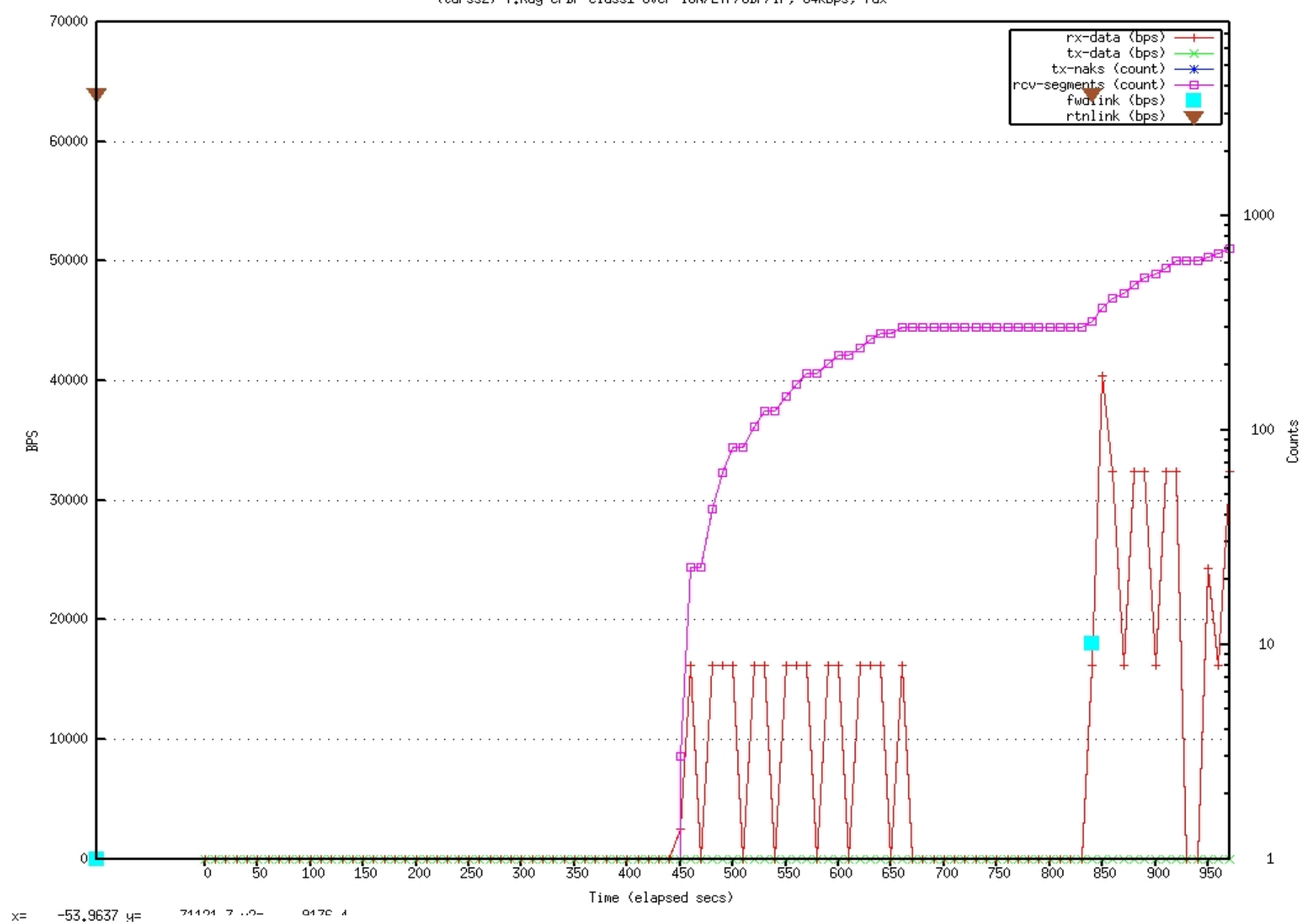

Figure 7: Sample result from ION-TDRSS Demonstration

A retest was done in the CSTL lab to try to reproduce the LTP state machine problem that occurred during the TDRSS ION testing. The retest used CFDP Class 1 over ION (BP/LTP/UDP/IP) which is identical to the protocol stack from previous TDRSS ION testing. This retest was done using a hard-line connection and the anomaly could not be recreated. During the retest depicted in Figure 8 the forward link is disabled for one, two, three, and ten minutes. Each time LTP transfer resumes after each interruption. The test begins with the forward link down from 70 seconds to approximately 120 seconds. The LTP state machine problem is not seen during this series of events.

\section{Summary}

Earth Science and LEO missions have some unique characteristics like simplex and asymmetric link rates. An internetworking protocol that could handle these characteristics would be advantageous to earth science missions. Missions would be provided with the tools such as rate buffering, autonomous routing, and storing and forwarding to handle this dynamic environment. Additionally, the TDRSS ION test demonstrated the performance of file transfer (CFDP) over $\mathrm{BP} / \mathrm{LTP} / \mathrm{UDP} / \mathrm{IP} / \mathrm{AOS}-$ Encap protocols stacks satisfying several requirements in the CCSDS DTN in Space Green Book. [2] Some of the requirements of note are related to file transfer, simplex links, and Quality of Service. 


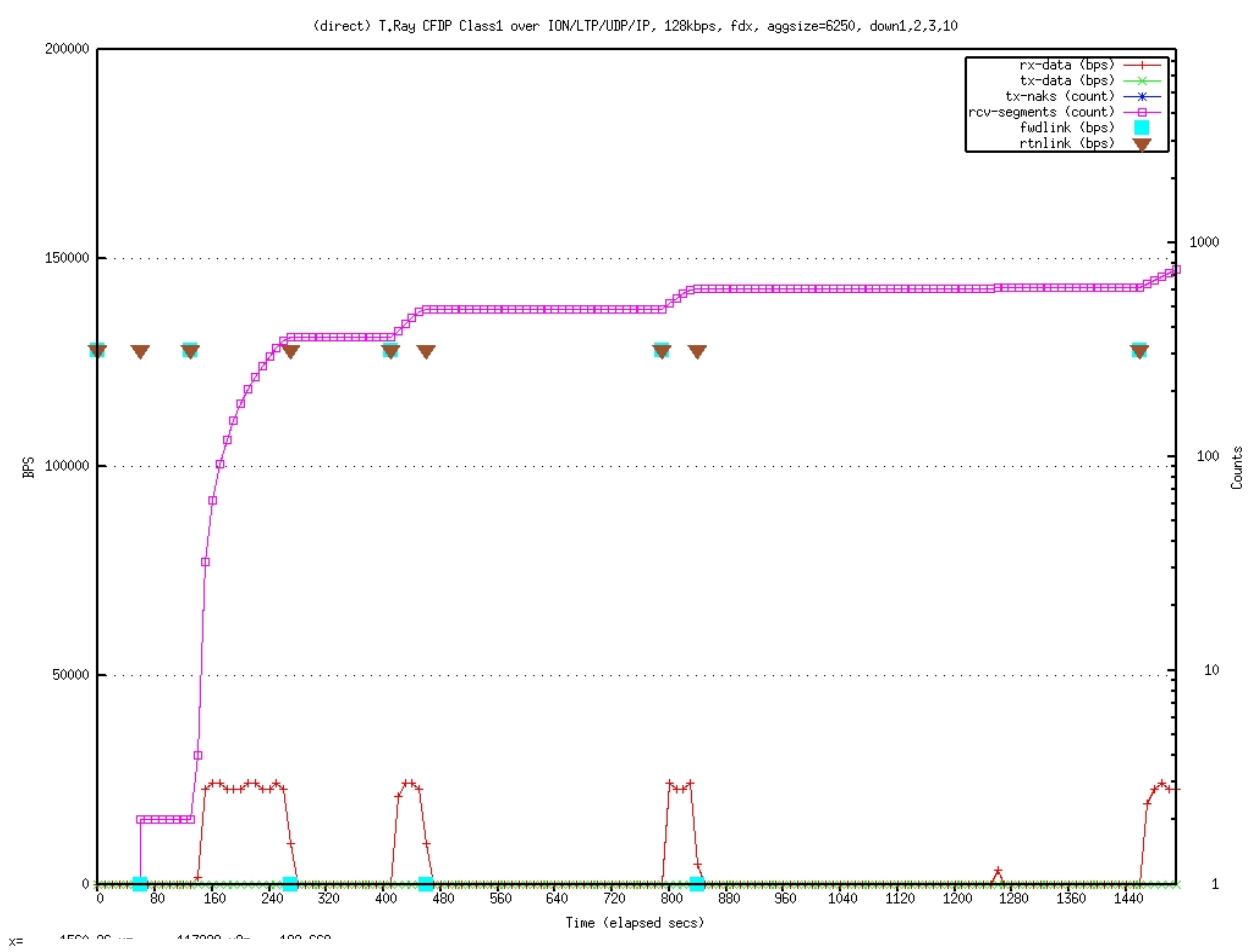

Figure 8: Sample output of ION-TDRSS retest

\section{Summary}

Collectively, the demonstrations provided empirical evidence that backs up claims that DTN can benefit an Earth Science Mission or any single flight and ground based system with satellite links. IRIS illustrated the potential for a reduction in latency as a result of a networked architecture and DTN usage in collaborative science scenarios. EO-1 showed the DTN store and forward function as an enabler for sensor webs and improved operations. Opportunities for collaboration or observation that were forgone as a result of the short response time needed could be taken advantage of with DTN usage. Using TDRS, a simple test showed DTN as a potential solution for issues surrounding asymmetric links and disparate space/ground links. Custody transfer enabled file capture on the ground that would not be possible with today's tools. And as is seen quite often, indirect benefits are uncovered, as was the case with the EO-1 recovery of housekeeping data. Operations and ground station infusion provided valuable feedback for future mission infusion. While much was learned with these demonstrations, further investigation will be needed to fully understand the benefits of DTN to Earth Science missions and space internetworking.

\section{FUTURE WORK}

Earth Science Missions are increasing the amount of science data being returned to Earth constantly. Decadal Survey missions[3] will have hundreds of gigabits of data per day that must be down linked. Further testing should be done to observe the DTN protocol working in mission like scenarios at higher data rates. Analysis should be done to determine challenges and benefits associated with DTN protocols at higher data rates.

\section{REFERENCES}

[1] National Aeronautics and Space Administration, "The New Age of Exploration, NASA's Direction for 2005 and Beyond," National Aeronautics and Space Administration, NP-2005-01-397-HQ, Washington, DC, Feb. 2005.

[2]The Consultative Committee for Space Data Systems, "Rationale, Scenarios, and Requirements for DTN in Space", Informational Report, CCSDS 734.0-G-1, Green Book, August 2010.

[3] Earth Science and Applications from Space: National Imperatives for the Next Decade and Beyond (Committee on Earth Science and Applications from Space: A Community Assessment and Strategy for the Future, National Research Council, 2007). 


\section{BIOGRAPHIES}

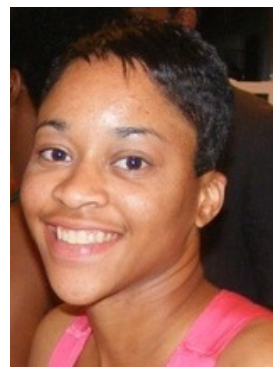

Faith A. Davis is a communications engineer in the Microwave and Communication Systems Branch at NASA Goddard Space Flight Center. She received a B.S.E.E. from Howard University in 2005 and a M.S.E.E. from Stanford University in 2007. Prior to joining NASA she worked as a communications engineer at Lawrence Livermore

National Laboratory in Livermore, CA. Since joining GSFC she has been involved in technology development, prototyping, and testing in support of the Delay Tolerant Networking Readiness Project. She has also supported the branch by providing her knowledge of networking and other higher layer stack functions.

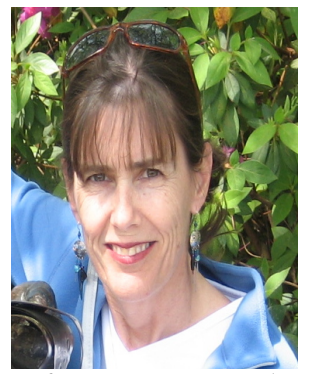

Jane K. Marquart is a senior software systems engineer in the Flight Software Branch at the NASA GSFC. She started her career working on the IUE spacecraft in 1979 and has held a variety of both ground and flight software positions for GSFC missions since, including the software systems lead on the WMAP spacecraft. Since 2001, Ms. Marquart has been involved in technology development and prototyping for both the flight software branch and the CCSDS Standards program. Ms. Marquart has a Bachelors degree from the University of Maryland, College Park. Go Terps!

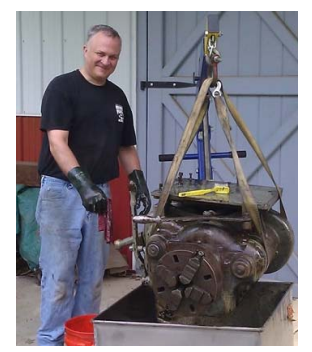

Greg D. Menke is a flight software engineer in the Flight Software Branch at the NASA GSFC. He has worked at GSFC since 2000, coming from a background in hard \& soft realtime embedded system software engineering, financial systems, industrial controls and network engineering. Mr. Menke has a Bachelors degree from the University of Maryland, Baltimore Campus. In his spare time, he volunteers in several industrial history preservation efforts. 